\title{
Derecho a la información en Coahuila: Una perspectiva desde la justicia constitucional ${ }^{1}$
}

\author{
Right to information in Coahuila: A perspective from the constitutional justice
}

José Luis, Leal Espinoza $^{2}$
Universidad Autónoma de Coahuila, México
(iD https://orcid.org/0000-0002-9072-0057
doi) $h$ https://doi.org/10.29105/pgc6.12-11

\section{RESUMEN}

Este artículo, a modo de ensayo, tiene como objetivo desarrollar una propuesta de comprensión de la integración Los artículos 46, de la Ley de Acceso a la Información Pública y el 9, bis de la Ley de Notariado de Coahuila, establecen como sujetos obligados indirectos a los Notarios Públicos, en razón de que éstos llevan a cabo actos de autoridad. En el objetivo general se establecieron las pautas que el legislador determinó los criterios que se cumplieron en las obligaciones en materia de transparencia. Las razones expresadas en la resolución se expusieron a través de los métodos documental y cualitativo, bajo el cual el juez constitucional instructor del amparo indirecto 186/2016, establece que, contrario a lo expresado por el legislador, al notario no puede considerársele como sujeto obligado indirecto, toda vez que esta figura no encuadra en ningún supuesto relacionado con la coadyuvancia al auxilio o colaboración de las entidades públicas, ejercicio del gasto público, utilizar o disponer de recursos públicos, subsidios o estímulos fiscales, esta razón de forma es entendida en el sentido de que no recibe un sueldo del Estado. cia.

Palabras clave: Derecho a la información, justicia constitucional, políticas públicas, ponderación, transparen-

\section{ABSTRACT}

Articles 46, of the Law of Access to Public Information and 9, bis of the Coahuila Notary Law, establish that public Notaries are indirect obligated subjects, because they carry out acts of authority. The general objective established the guidelines that the legislator determined the criteria that were met in the transparency obligations. The reasons expressed in the resolution were set forth through the documentary and qualitative methods, under which the constitutional examining judge of indirect amparo 186/2016, establishes that, contrary to what is expressed by the legislator, the notary cannot be considered an obligated indirect subject, since this figure does not fit into any assumption related to aid to or assistance from public entities, exercise of public spending, use or disposition of public resources, subsidies or fiscal stimuli, this form reason is understood in the sense that you do not receive a salary from the State.

Keywords: Constitutional justice, public policies, right to information, transparency, weighting.

Recibido: 02 de Noviembre 2019 - Aceptado: 04 de Marzo 2020 - Corregido: 04 de Mayo 2020

Cómo referenciar este artículo:

Leal Espinoza, J. L. (2020). Derecho a la información en Coahuila: Una perspectiva desde la justicia constitucional Politica Globalidad y Ciudadanía, 207-224. Recuperado de http://revpoliticas.uanl.mx/index.php/RPGyC/ article/view/155

\footnotetext{
1 Proyecto de investigación "Derecho a la información en Coahuila: una perspectiva desde la justicia constitucional", Financiado por Universidad Autónoma de Coahuila

2 Doctor en Derecho Constitucional y Derechos Fundamentales por la Universidad de Alicante, España. Coordinador de Posgrado e Investigación de la Facultad de Ciencias Políticas y Sociales, Universidad Autónoma de Coahuila. Email: jose.leal@uadec.edu.mx
} 


\section{1.- INTRODUCCIÓN}

La sentencia motivo de controversia estipula que, si bien la figura del notario público coadyuva en auxilio o colaboración de entidades públicas, no realiza actos de autoridad en sentido estricto, pues el notario público no forma parte de la administración pública centralizada, desconcentrada o descentralizada ni es parte de los poderes del Estado. Por tal motivo, su función pública únicamente va dirigida a autentificar hechos o actos jurídicos con fuerza de fe pública frente a todos. Dicho lo anterior, la problemática consiste en verificar si las obligaciones enlistadas para los notarios como sujetos obligados indirectos en las normas impugnadas, vulneran o no el marco constitucional y legal en materia de transparencia y protección de datos personales.

El documento se estructura en cinco partes. En la primera sección se formulará un estudio integral sobre las razones que tuvo el Poder Constituyente permanente en replantear el modelo nacional en materia de transparencia, poniendo especial énfasis en la nueva categoría de sujetos obligados indirectos establecidos en la reforma constitucional al artículo $6^{\circ}$ constitucional, así como el renovado marco competencial que otorga a las Entidades Federativas en la materia.

A la luz de lo anterior, se realiza un estudio constitucional sobre la pertinencia de la figura legal introducida por el legislador de Coahuila, al contemplar al notario como sujeto obligado indirecto en materia de transparencia, exponiendo las razones de índole legislativo y jurisprudencial que justifican la posición discrepante con el criterio judicial primigenio. Una vez superada la fase anterior, las siguientes dos secciones explican que la naturaleza de la información regulada por la ley como obligatoria a la luz del marco normativo constitucional y legal en materia de derecho a la información y protección de datos personales.

El objeto específico del cuarto apartado consiste en demostrar que cada una de las obligaciones consagradas en la Ley de Acceso a la Información y Ley de Notariado respectivamente, no vulneran los derechos de particulares en lo que respecta al derecho humano a la protección de datos personales, toda vez que no se configura dentro de ninguna de las hipótesis que consagra la propia ley como información confidencial, al tratarse de versiones públicas que armonizan los principios constitucionales de transparencia e intimidad. Precisamente, la última sección de la presente comunicación concluye en este sentido, ya que al realizar un test de proporcionalidad al caso concreto, se concluye que ningún contenido esencial resulta vaciado por completo, ya que los aludidos principios resultan optimizados, resultando ello en una interpretación contraria a la sostenida originalmente por el juez de distrito.

\section{2.- FUNDAMENTO TEÓRICO}

\section{La reforma constitucional en materia de transparencia y los nuevos sujetos obligados}

La reforma constitucional en materia de transparencia y acceso a la información (Leal y López,

Revista Política, Globalidad y Ciudadanía, Vol. 6. No. 12, Julio - Diciembre 2020, Universidad Autónoma de Nuevo León, Monterrey, México, ISSN 2395-8448. 207-224. http://revpoliticas.uanl.mx/index.php/RPGyC/article/view/155 
2019) a los artículos 6, apartado a), y 116, fracción VIII, estableció un nuevo catálogo de sujetos obligados en la materia. En el Dictamen formulado por la Cámara de Senadores, realizado posterior a la presentación de las tres iniciativas destaca de manera trascendental el reconocimiento de los particulares como nuevos sujetos obligados en el contexto del nuevo marco constitucional propuesto por el Constituyente permanente (las cursivas son nuestras), así como la cita del Código de Buenas Prácticas elaborado por encargo del antiguo IFAI.

Se está proponiendo que los particulares que actúen como autoridad o la auxilien, o aquéllos que ejerzan gasto público, quedan -por esos simples hechos o circunstancias- de forma automática o lógica como sujetos obligados -indirectos- en materia de acceso a la información pública. Sin dejar de advertir, que respecto a la información pública de aquéllas personas físicas o morales (jurídicas-colectivas) que reciban recursos públicos, o realicen actos de autoridad, se propone que el acceso se realice a través de la autoridad, ente, órgano u organismo público que hubiere entregado los montos respectivos, o sea el que supervise la actuación de esos particulares; es decir, en este caso, se está proponiendo que las personas físicas o morales referidas sean sujetos obligados indirectos en el acceso a la información pública, siendo el vehículo para ello los propios sujetos obligados directos respectivos. (Gaceta del Senado de la República, 2012, p. 54)

Otra cuestión ampliamente debatida fue la inclusión, como sujetos obligados, de personas privadas. Cabe recordar que 23 leyes estatales establecen que las personas físicas y morales que reciben recursos públicos o realizan funciones públicas son sujetos obligados directos del derecho de acceso a la información. La experiencia muestra que esta práctica presenta importantes problemas jurídicos y prácticos. No obstante lo anterior, es cierto que existe una cantidad importante de información relevante que se encuentra en posesión de particulares y de organizaciones privadas. En atención a los múltiples comentarios de las reuniones regionales sobre la importancia de garantizar el acceso a esta información, el CBP propone, retomando a la jurisprudencia de los tribunales federales, que cuando un particular realiza actos de autoridad, la información que genere será considerada como información pública. Este artículo permite otorgar así acceso a la información de los sindicatos, las asociaciones empresariales o los concesionarios de un servicio público respecto de los actos que realicen en calidad de autoridades. Debe hacerse notar, sin embargo, que se propone que el acceso no se dará de manera directa, sino a través de la autoridad que supervise la actuación de esos particulares. La razón de esta disposición atiende a razones prácticas y a evitar cargas regulatorias excesivas o incumplibles para las personas privadas." (Ibid, p.57)

Particulares que reciben recursos públicos o ejercen actos de autoridad: En relación con la información de particulares el citado Código de Buenas Prácticas, plantea que los particulares 
que ejercen actos de autoridad estarán obligados a entregar la información relacionada con esos actos a través del sujeto obligado que supervise estás actividades. Siendo que lo importante a destacar es que cuando un particular realiza actos de autoridad, la información que genere sea considerada como información pública.

Además, en el Código de Buenas Prácticas, se expone que "por acto de autoridad debe entenderse, de acuerdo con la jurisprudencia del Poder Judicial de la Federación, un acto unilateral, coercitivo y con imperio que crea, modifique o extinga situaciones jurídicas que afecten la esfera legal de los gobernados.

También, se expone que a fin de regular el derecho de acceso a la información pública que pudieran poseer los particulares se atendió a la ampliación del concepto de autoridad que para efectos del amparo ha sostenido el Poder Judicial de la Federación: son autoridad aquellos individuos que ejerzan actos unilaterales y usen la fuerza pública para alterar la esfera jurídica de los gobernados. Es decir, la autoridad se distingue por el hecho de que realiza actos con imperio, más que por los sujetos que ejecutan el acto. Ahora bien, ante una administración pública cada día más compleja, la distinción del carácter de cada uno de los actos realizados por entes públicos o que los mismos delegan incluso a particulares se hace más difícil. Así, por ejemplo, casos como PEMEX o Seguro Social en ciertos esquemas actúa con imperio y por tanto es una autoridad y en otros no. Véase por ejemplo la tesis confederación deportiva mexicana, asociación civil. Tiene la calidad de autoridad para efectos del juicio de amparo, cuando sus actos se funden en la ley general del deporte, en relación con su estatuto. Es importante precisar que el acceso a la información de estos particulares, en su caso, se limita a aquella información relacionada con sus funciones equiparables a actos de autoridad y no al conjunto de sus actividades. A mayor abundamiento de lo anterior, cabe recordar, que la fracción VI del artículo $6^{\circ}$ de la Constitución Política de los Estados Unidos Mexicanos establece que "las leyes determinarán la manera en que los sujetos obligados deberán hacer pública la información relativa a los recursos públicos que entreguen a personas físicas o morales”. Esta disposición constitucional deja justificado claramente buena parte de la propuesta que se plantea en la presente iniciativa.

En efecto, hay que reconocer que existen algunas personas de carácter privado, ya sean físicas o morales, que ejercen funciones públicas, ya sea por delegación o por un mandato legal.

Como atinadamente apunta Sergio López Ayllón, “este es el caso de los notarios públicos, de los concesionarios de bienes o servicios públicos, de los colegios de profesionistas o cámaras comerciales o industriales y aun de los sindicatos. La pregunta es si éstos son sujetos obligados del derecho de acceso a la información y, si esto es así, cuáles son los mecanismos para ejercerlo". Más aún, el problema se plantea respecto de las personas privadas que, cada vez con mayor frecuencia, desarrollan funciones públicas o ejercen por delegación o mandato legal funciones 
de autoridad, sin constituirse propiamente como entidades públicas. La calidad de sujetos pasivos del derecho de acceso a la información de estas personas plantea cuestiones complejas, en particular respecto a los procedimientos para hacer efectivo el derecho.

Dejando claro, que no por recibir recursos del erario o por ejercer una parte del gasto estatal, toda actividad de estas personas se convierte en materia de acceso público, sino únicamente de aquellas en lo que se aplicó el recurso público o bien se ejercitó o ejecuto un acto de autoridad, no así respecto de otras distintas a estos supuestos, no pudiendo entender que todas las actividades serán consideradas como parte del acceso a la información, sino solo en los casos ya descritos, lo anterior con el fin de no afectar la privacidad e intimidad de las personas.

Los anteriores razonamientos legislativos contienen claramente el espíritu del Poder reformador de la Constitución (Asensi, 1998), al establecer de manera precisa que los particulares que ejercen actos de autoridad también pueden ser sujetos obligados indirectos en materia de transparencia, dejando incluso la potestad a las Entidades Federativas de acuerdo al numeral 116, fracción VIII, de garantizar el derecho de acceso a la información y protección de datos personales en posesión de los sujetos obligados, de acuerdo a los principios del artículo $6^{\circ}$, apartado a).

Por ello, es factible y acorde a una interpretación conforme con el artículo $6^{\circ}$ constitucional, considerar como sujetos obligados indirectos a los notarios públicos, toda vez que el concepto material que tiene el legislador al momento de introducir la figura en el texto fundamental es acorde a las nuevas realidades derivadas de la teoría contemporánea de la autoridad, que retoman incluso del Código de Buenas Prácticas el concepto ya ampliamente difundido tanto en la doctrina, la legislación y la jurisprudencia del Poder Judicial de la Federación, es decir “un acto unilateral, coercitivo y con imperio que crea, modifique o extinga situaciones jurídicas que afecten la esfera legal de los gobernados".

\section{El Notario como sujeto obligado indirecto y su discrepancia en el caso concreto}

Sentado lo anterior, es menester hacer énfasis en las dos categorías delineadas por el juzgador en el juicio de amparo indirecto sometido a debate en el presente amparo en revisión, ya que en la resolución objeto de controversia se establece claramente que al notario público no puede considerársele como autoridad en sentido estricto, ya que la información que posee no es pública. Lo anterior, se realiza con base en un ejercicio argumentativo de dos dimensiones (Atienza, 2013): que el derecho a la información es una garantía individual y social, y que la transparencia y publicidad de los actos de gobierno son inherentes (Pleno SCJN, 2013, p.5). La siguiente premisa de la cual parte la negativa es que los notarios no son funcionarios públicos, ya que no están sujetos a los derechos y deberes de los funcionarios oficiales, ni el Estado responde por 
sus actos. (Contradicción de Tesis 24/2003-SS, p. 70)

No obstante lo anterior, a pesar de que la Segunda Sala de la Suprema Corte emitió criterio en el sentido de que los notarios no eran autoridad para los efectos precisados, también lo es que en esa misma decisión se desprende el reconocimiento de que, a pesar que los notarios no son funcionarios públicos, los mismos "sí realizan una función pública, ya que autentica hechos o actos jurídicos con fuerza de fe pública frente a todos, incluyendo al Estado; además instruye a los particulares que a él acuden del alcance jurídico de esos actos, resguarda documentos originales y expide copias" (Contradicción de Tesis 24/2003-SS, p. 70). Precisado esto, es indispensable formular las siguientes interrogantes:

- ¿El carácter de sujeto obligado indirecto con el que se identifica a los notarios en las leyes impugnadas los convierte automáticamente en autoridad o en funcionarios públicos?

- ¿Las obligaciones para los sujetos indirectos en materia de transparencia sólo van dirigidas a quienes reciben presupuesto público o a quienes ejercen actos de autoridad "en sentido estricto"?

Respecto a la primera cuestión, es dable afirmar que, de acuerdo a lo expuesto por la autoridad responsable existe un vicio lógico de petición de principio cuando se contesta la primera interrogante, ya que de manera tajante se concluye que el notario no es autoridad porque no es un funcionario público, aunque su función en sí sea pública. Esto no es más que exponer las mismas razones de manera circular, dejando segmentado el debate principal, toda vez que el tema de fondo consiste en indagar si bajo el contexto de las nuevas obligaciones en materia de transparencia establecidas en la Constitución y la Ley del Estado de Coahuila, el notario realiza o no actos de autoridad, siendo en consecuencia así un sujeto obligado indirecto.

La segunda cuestión es respondida por la autoridad responsable al afirmar que a pesar de que los notarios públicos ejercen una función pública, las obligaciones de transparencia únicamente tienen como destino los actos de gobierno, a partir del esquema de rendición de cuentas, es decir, manejo de recursos públicos, satisfacción de los servicios a su cargo y el cumplimiento de funciones públicas. En la sentencia, si bien se realiza un estudio parcial basado en la Exposición de motivos de la reforma constitucional en materia de transparencia, este resulta incompleto, ya que para saber si las obligaciones en materia de transparencia también incluyen a los notarios públicos es obligatorio realizar un estudio sobre la naturaleza propia de la información generada por estos en el ejercicio de su función, es decir, un análisis que no solamente se limite al aspecto formal, sino material, explicando a detalle si la generación de esa información es suficiente para ser considerada como de naturaleza pública.

Dicho en otras palabras, la Constitución en su artículo $6^{\circ}$ establece un nuevo catálogo de sujetos obligados indirectos abrió la posibilidad situándose en un paradigma distinto bajo el cual 
lo analiza la autoridad responsable, es decir, el primer modelo de transparencia en su primera fase 2002, con la entrada en vigor de la Ley Federal de Transparencia y Acceso a la Información Gubernamental y la correlativa reforma constitucional de 2007 (Islas, 2007, pp. 155-174) , estableció un diseño preliminar mediante el cual se le reconoció el carácter tanto individual como social a este derecho fundamental, así como a la protección de la vida privada y datos personales (Carbonell, 2008).

Sin embargo, el esquema a través del cual se razona en la decisión reclamada parte de un modelo en el cual únicamente la autoridad formalmente reconocida por las leyes es aquella que puede ser sujeta de obligaciones en materia de transparencia. Por ello, resulta válido afirmar que existen dos elementos que ha soslayado la autoridad judicial. El primero está relacionado con la existencia de sujetos obligados indirectos distintos a las autoridades desde el plano formalmente legal, es decir, determinar si los notarios públicos en el ejercicio de una función pública realizan actos de autoridad, y además, establecer si es constitucional y legalmente válido que las Entidades Federativas en el ejercicio de su libertad configurativa contemplen esta posibilidad.

Un cambio de paradigma trascendental en los últimos años ha sido la mutación legislativa que ha sufrido el concepto de autoridad para efectos del juicio de amparo (Sartori, 2016). En la Ley de Amparo vigente el legislador ha dado un viraje importante, al establecer esta nueva posibilidad. En la Exposición de motivos de dicha legislación y después de un análisis reflexivo sobre las distintas vías (civil, administrativa) que pudieran ejercitarse para hacer valer los derechos, el legislador determinó que el juez deberá hacer caso a la afectación material del derecho fundamental en cuestión, sobre el acto de autoridad, así como las condiciones del particular para defenderse, y no tanto al aspecto formal de quien emite el acto violatorio de derechos.

... existen casos en los que deban tener este cauce procesal para obtener una protección más eficaz, más justa y por tratarse de una violación directa a este tipo de derechos. Por ello se considera que la mejor forma de plasmar este concepto en esta Ley y de explicarlo en el presente dictamen es definir el acto de autoridad y que los particulares tendrán dicha calidad cuando sus actos u omisiones sean equivalentes a los de autoridad, que afecten derechos en los términos del acto de autoridad que objetivamente se define en la fracción II, y cuya potestad o función derive de una norma general y abstracta (Gaceta del Senado de la República, 2011, p. 21).

Es decir, el concepto de personas físicas que realizan "actos de autoridad" como sujetos obligados en la Ley General establecido en el artículo 23 no es un catálogo cerrado a solamente aquellas personas que reciban recursos públicos toda vez que el propio legislador federal determinó: “en los ámbitos federal, de las Entidades Federativas y municipal”. Es decir, se debe atender al ejercicio material de ese "acto de autoridad" para determinar si realmente el ejercicio de la función notarial deviene en la generación de información de interés público, y así concluir 
si el ejercicio del derecho a la información pública se debe proteger en las relaciones generadas entre los notarios y la sociedad (Sala SCJN, 2012, p. 798).

Para lograr lo anterior, es necesario partir de una definición mínima de información de interés público que brinda la propia Ley de Acceso a la Información en su numeral 3, fracción XII, como: "la información que resulta relevante o beneficiosa para la sociedad y no simplemente el interés individual, cuya divulgación resulta útil para que el público comprenda las actividades que llevan a cabo los sujetos obligados". Es decir, la información que están obligados los notarios a dar publicidad que refieren los dos artículos impugnados debe fundamentarse no sólo en las razones del legislador local en el ámbito de su libertad configurativa como Entidad Federativa, sino que la propia información debe satisfacer el canon de "interés público", además de ser proporcional y útil en el marco de la sociedad democrática.

La información a que se refieren las normas impugnadas es de interés público por su propia naturaleza y es generada por los notarios en el contexto de su función pública

Tal y como quedó apuntado, en la resolución se evade el problema de fondo al afirmar que la información a que se refieren los artículos 46 de la Ley de Acceso a la Información Pública y Protección de Datos Personales para el Estado de Coahuila de Zaragoza, y el 9, bis de la Ley del Notariado de esa misma entidad, no es de interés público porque quien la emite no es una autoridad en sentido estricto. Este razonamiento es circular y redundante, toda vez que, en la propia sentencia se reconoce que el notario desempeña una función pública, pero el carácter de la información en ningún momento es analizado en la resolución, toda vez que para ello hubiera sido necesario estudiar la naturaleza material de la información sometida a debate.

Por tanto, en primer término serán analizadas las razones expuestas por el legislador del Estado de Coahuila en la Exposición de Motivos de las legislaciones objeto de controversia, seguidas del razonamiento lógico jurídico enlazado con criterios jurisprudenciales que explicarán a detalle que la información que como sujetos obligados están obligados los notarios a dar publicidad es de interés público, a través de un método hermenéutico (López, 2016, pp. 11-12) integral del contexto de la reforma legal al sistema estatal de transparencia y gobierno abierto de esta Entidad. Las normas impugnadas establecen que la información que están obligados los notarios a publicitar es la siguiente:

- Difundir u nombre, domicilio, teléfono oficial y número de fiat notarial;

- Publicar los servicios que ofrece y su costo;

- Divulgar la plantilla de personal y tipo de seguridad social que se le ofrece;

- Publicar los índices de protocolo; V. Difundir las versiones públicas de las actas fuera de protocolo;

Revista Política, Globalidad y Ciudadanía, Vol. 6. No. 12, Julio - Diciembre 2020, Universidad Autónoma de Nuevo León, Monterrey, México, ISSN 2395-8448. 207-224. http://revpoliticas.uanl.mx/index.php/RPGyC/article/view/155 
- Divulgar la relación de cursos o programas de actualización o capacitación que se realicen y los que se ofrezca al personal; y

- Un listado con el número de recepción o de identificación que entregue la autoridad fiscal, de aquellas retenciones enteradas ante la misma.

En la Exposición de motivos de la Ley de Acceso a la Información se establece que:

A fin de atender a los principios del derecho a la información, la Información Pública de Oficio, reconoce distinciones entre los Sujetos Obligados, de acuerdo a la naturaleza y circunstancias de cada uno. Es por ello, que precisa para aquellos que se definen como Entidades Públicas: Poder Ejecutivo, Poder Legislativo, Poder Judicial, Ayuntamientos, Universidades e Instituciones de Educación Superior Pública, Sistemas de Agua y Saneamiento, Organismos Constitucionalmente Autónomos y Fideicomisos y Fondos Públicos, obligaciones generales y especiales; y aquellos Sujetos Obligados que no son Entidades Públicas: Partidos Políticos, Sindicatos, Organizaciones de la Sociedad Civil e Instituciones de Beneficencia, sólo poseen obligaciones especiales.

Dicha distinción entre Sujetos Obligados, permite garantizar el derecho a la información y la protección de los datos personales, atendiendo, como se ha hecho mención, no sólo a la fiscalización de recursos públicos, sino al interés público de conocer información que propicia o retarda el desarrollo del Estado.

En este sentido, la diferenciación normativa que hizo el legislador respecto de los sujetos obligados directos e indirectos es patente, ya que los notarios públicos (siguiendo la propia exposición de motivos) pertenecen a esta última categoría debido a que su función es esencial para el Estado y de interés público. Por tanto, el legislador en este caso en particular decidió que:

A cada uno de ellos, dependiendo de sus funciones e interacción con la comunidad, se le determinan obligaciones de difundir cierta información relacionada con su labor pública.

Por tales motivos, y siguiendo esta línea argumentativa se desprende que existe una distinción realizada por el legislador al momento de definir a los "sujetos obligados" y los "sujetos obligados indirectos", es decir, corresponde a los primeros las obligaciones que se detallan en los artículos 4 a 12, mientras que para los "sujetos obligados indirectos" corresponden únicamente las obligaciones específicas que se detallan en los artículos 43 a 49, dependiendo de quien se trate. Es decir, se les debe atribuir únicamente las "Disposiciones Generales" establecidas en el Capítulo Tercero, Sección Primera, de la Ley de Acceso a la Información, es decir, las relativas a la información pública de oficio, mismas que se encuentran establecidas en los numerales 17 a 20 del mencionado dispositivo legal, es decir:

“Artículo 17. Con excepción de la información reservada o confidencial prevista en esta ley, los sujetos obligados deberán difundir, actualizar y poner a disposición del público de manera 


\section{6}

proactiva, la información pública a que se refiere este capítulo.

Artículo 18. Las páginas electrónicas utilizadas por los sujetos obligados para la difusión de información pública, observarán los siguientes lineamientos:

I. La página de inicio tendrá un vínculo de acceso, fácilmente identificable y accesible que cumpla con los requerimientos de sistematización, comprensión y organización, directo a donde se encuentre la información pública a la que se refiere este capítulo;

II. La información que se difunda en las páginas electrónicas deberá ser confiable, completa y oportuna;

III. El lenguaje utilizado será claro, sencillo, accesible y que facilite la comprensión de las personas que consulten dichas páginas;

IV. Deberán contar con un teléfono de atención y correo electrónico por medio del cual los ciudadanos puedan realizar opiniones, quejas, o sugerencias que atienda directamente el órgano de control interno o equivalente; $y$

V. Deberán de utilizarán formatos abiertos y de fácil comprensión. El instituto establecerá los lineamientos necesarios para asegurar que la información cumpla con lo dispuesto en este artículo.

Artículo 19. La información pública de oficio deberá de actualizarse en los medios electrónicos disponibles por lo menos una vez al mes. En todos los casos se deberá de indicar en el medio electrónico la fecha de actualización por cada rubro de información.

Artículo 20. El sujeto obligado deberá difundir, dentro del primer mes de cada año, un calendario de actualización de la información, por cada contenido y área responsable.

Para la publicación de la información pública de oficio, los sujetos obligados utilizarán formatos de fácil comprensión y deberá de tener disponible su traducción en lenguas indígenas.

Dicho esto, la Sección Segunda del Capítulo Tercero de la Ley de Acceso a la Información corresponde únicamente a las entidades públicas, en sentido contrario a lo determinado erróneamente por el Juez en la resolución de amparo indirecto, al pretender atribuirle obligaciones que no corresponden al notario público, descontextualizando así la cabal y correcta comprensión del numeral 46 tildado de inconstitucional.

En síntesis, los artículos 17 a 20 de la Ley de Acceso a la Información establecen el medio o instrumento (medios digitales) a través del cual se garantiza que la información catalogada como pública de oficio respecto de los sujetos obligados indirectos (en este caso los notarios) sea realmente efectiva, es decir, esté al alcance de cualquier persona en la Entidad (artículo 46).

En sintonía con ello, la proporcionalidad (Zagrebelsky, 2003) del medio empleado debe perseguir así una finalidad constitucionalmente legítima, ser adecuada, necesaria y útil para el fin que se propone. Para lograr tal propósito, el test de proporcionalidad (Alexy, 1991) que se

Revista Política, Globalidad y Ciudadanía, Vol. 6. No. 12, Julio - Diciembre 2020, Universidad Autónoma de Nuevo León, Monterrey, México, ISSN 2395-8448. 207-224. http://revpoliticas.uanl.mx/index.php/RPGyC/article/view/155 
realice en el caso concreto mediante un control abstracto de la norma (amparo contra leyes) deberá verificar estas tres fases o pasos. Este ejercicio hermenéutico será realizado en la siguiente sección, con el fin de verificar la legitimidad constitucional de las normas objeto de debate.

Legitimidad de la medida impuesta a los Notarios como sujetos obligados indirectos de difundir la información pública de oficio establecida en el artículo 46 de la Ley de Acceso a la Información

El test de proporcionalidad propuesto en el caso concreto consiste en primer lugar, en determinar si los artículos 46, de la Ley de Acceso a la Información Pública y Protección de Datos Personales para el Estado de Coahuila de Zaragoza, y el 9, bis de la Ley del Notariado de esa misma entidad, persiguen una finalidad constitucionalmente legítima. En este sentido, dicha finalidad puede ser medida objetivamente a partir de lo dispuesto por el artículo 2, fracción XII, de la Ley de Acceso a la Información (vigente al momento de la presentación de la demanda), la cual dispone que la información pública es toda información en posesión de los sujetos obligados, con excepción de la que tenga el carácter de confidencial, misma definición que es similar a la dispuesta por la Ley Federal de Acceso a la Información, vigente al momento de la demanda o a la actual Ley General de Acceso a la Información.

Por ello, mediante un ejercicio de subsunción de lo dispuesto por los artículos 58 y 59 de esa misma Ley, se concluye que la información que tiene el carácter de reservada, cuando así se dicta por una autoridad por razones de interés público por las razones que se exponen enseguida:

- Ponga en riesgo la vida, la seguridad o la salud de cualquier persona;

- Pueda comprometer la materia de seguridad pública del Estado y sus Municipios;

- Dañe la estabilidad económica y financiera del Estado;

- Ponga en riesgo la implementación, administración y seguridad de los sistemas de datos personales;

- Aquella cuya divulgación pueda causar un serio perjuicio a: las actividades de prevención o persecución de los delitos; administración de justicia o la seguridad de un denunciante, querellante o testigo, así como sus familias, en los términos de las disposiciones aplicables; recaudación de las contribuciones; y la que contengan las opiniones, recomendaciones o puntos de vista que formen parte del proceso deliberativo de los servidores públicos, hasta en tanto sea adoptada la decisión definitiva, la cual deberá estar documentada; y

- Cuando se trate de información de estudios y proyectos cuya divulgación pueda causar un daño al interés del Estado o suponga un riesgo para su realización. 
- Expedientes de averiguaciones previas, siempre y cuando no se haya determinado el ejercicio de la acción penal o el no ejercicio de la misma, tiempo en el cual serán disponibles en versión pública en términos de las disposiciones aplicables;

- Acuerdos y procedimientos de mediación, negociación, arbitraje y conciliación hasta en tanto no se tome un acuerdo firme;

- Expedientes judiciales o de los procedimientos administrativos seguidos en forma de juicio en tanto la sentencia no haya causado ejecutoria, posteriormente serán accesibles en su versión pública en términos de las disposiciones aplicables; y

- Expedientes de las denuncias y procedimientos de juicio político y de declaración de procedencia en materia de responsabilidad penal.

Ahora bien, el carácter de información reservada queda dispuesta por los diversos numerales 67, 68 y 69 de la Ley de Acceso a la Información, donde se dispone que tiene dicha naturaleza cuando se trate de la vida privada y los datos personales, la cual merece un tratamiento especial de parte de aquellos servidores públicos que la reciban, gestionen, administren o resguarden, dichos supuestos son los siguientes:

- Los datos personales que requieran del consentimiento de las personas para su difusión, distribución o comercialización y cuya divulgación no esté prevista en una ley;

- La protegida por los secretos comercial, industrial, bancario, fiscal, fiduciario, médico y profesional; y

- La información protegida por la legislación en materia de derechos de autor o propiedad intelectual.

Idoneidad de la obligación de difundir la organización interna de la función de la institución notarial

En consideración de lo anterior, resulta pertinente analizar el catálogo de obligaciones que derivan de las normas objeto de impugnación, con el fin de verificar en la primera fase del test de proporcionalidad si éstas persiguen una finalidad constitucionalmente legítima en el marco de una sociedad democrática. Para lograr tal propósito, se ha propuesto por cuestión de método, sistematizar las obligaciones de la siguiente manera. Se analizarán en primer lugar aquellas obligaciones que derivan de la organización interna de la función de la institución notarial, estas son:

1. Difundir su nombre, domicilio, teléfono oficial y número de fiat notarial.

2. Publicar los servicios que ofrece y su costo.

3. Divulgar la plantilla de personal y tipo de seguridad social que se le ofrece.

4. Divulgar la relación de cursos o programas de actualización o capacitación que se reali- 
cen y los que se ofrezca al personal.

La función pública que desempeña un notario en su calidad de particular con autorización del estado es fundamentalmente de fe pública, la cual es entendida como "la facultad con la que están investidos determinados agentes para certificar que los hechos que les constan son verdaderos y auténticos" (Real Academia Española, 2016, p. 830), mientras que la fe pública notarial es "aquella que se otorga a través de la figura del notario y cuyos efectos jurídicos se traducen tanto en presunciones de veracidad e integridad como en juicios de legalidad, capacidad, y legitimación, que permiten operar en el tráfico jurídico respecto de cualquier operador” (Ídem).

La función de fe pública deriva directamente del mandato constitucional 121, el cual establece que "en cada Estado de la Federación se dará entera fe y crédito de los actos públicos, registros y procedimientos judiciales de todos los otros". Este concepto proviene originalmente de la Constitución Federal de 1824, cuando el Poder Constituyente originario tradujo la expresión "public acts" del sistema norteamericano refiriéndose a estos como a todas aquellas "manifestaciones de voluntad que conciernen a toda la comunidad y tienen a producir efectos jurídicos".

Posteriormente, con la promulgación de la Constitución de 1857, el Constituyente siguió la este mismo hilo conductor de la fe pública, y destacó su relevancia al grado que en 1870 fue presentado por los Estados de Hidalgo, Guanajuato y Aguascalientes, una propuesta de Ley Reglamentaria a este artículo que establecía que los documentos expedidos por las autoridades políticas, judiciales, municipales y militares, así como los registros y actos públicos de los notarios y escribanos, serían comprobados por sus respectivos gobernadores y, en su ausencia, por los presidentes de los tribunales superiores, además era necesario realizar una legalización, por medio de un timbre cuya forma reglamentaría el Ejecutivo de la República. (Del Pilar y Morales, 2015, p.22)

Derivado de lo anterior, es dable afirmar que la fe pública es una consecuencia directa de la obligación del Estado de preservar el orden y la seguridad jurídica, tan es así que ha sido preocupación desde el Constituyente originario de 1824. Por lo que el sistema de fe pública está envuelto en un contexto complejo de relaciones jurídicas que la mayor parte de los ciudadanos no puede presenciar, pero los actos necesitan verdad, es decir, ser creídos para ser aceptados, por lo que la función pública que lleva a cabo el notario debe ser transparente para abonar a la certeza y seguridad jurídica para con las personas que, si bien no presencian los actos, estos tengan confianza en la institución del notario mediante la propia apertura de la información que es de orden público.

Este último punto es quizá el punto medular del debate, el hecho que el acto no haya sido presenciado por la sociedad en general no significa que en automático convierta la información en reservada o confidencial, toda vez que el Estado tiene la obligación de supervisar esa función 
pública, por lo que en el marco de su ámbito competencial, en el caso concreto el Estado de Coahuila de Zaragoza, toda vez que no existe un marco referencial establecido a través de una Ley General por el Congreso de la República, cuenta con la facultad en ejercicio de las facultades residuales con base en el artículo 124 y en respeto al principio de reserva de ley, que se elaboró el paquete de reformas que ahora son objeto de debate.

En este contexto, que la sociedad coahuilense conozca como información pública de oficio el nombre, domicilio, teléfono oficial y número de fiat notarial, sus servicios así como su costo del cada notario que tiene a su cargo el ejercicio de la función pública en su Entidad, y la relación de cursos o programas de actualización o capacitación que se realicen y los que se ofrezca al personal (identificadas arriba como obligaciones 1, 2 y 4) es constitucionalmente legítimo, en razón de la fe pública que le es encomendada por el Estado, toda vez que la misma guarda una especial importancia debido a los valores y principios constitucionales de seguridad y certeza jurídica que derivan del bien constitucional, que es la fe pública que autoriza el Estado a los notarios, que si bien son particulares, el Estado autoriza como profesionales del derecho y son los ciudadanos acuden a los mismos para solicitar un servicio de fe pública, donde su el derecho fundamental patrimonial, la identidad, representación, entre otros, pudieran resultar visiblemente afectados en caso de un indebido actuar, por lo que la información previa que se pueda disponer del catálogo en la Entidad de notarios sobre esta obligación específica es fundamental para decidir con qué profesional acudir.

Respecto de la obligación concreta de: "divulgar la plantilla de personal y tipo de seguridad social que se le ofrece", es importante advertir que en este caso la finalidad que persigue la fracción referida es constitucionalmente legítima, toda vez que la misma no colisiona con la información considerada como confidencial, toda vez que no afecta a los particulares, entendidos estos como los trabajadores que laboran en la notaria respectiva, ya que, si bien los datos personales son definidos como "cualquier información concerniente a una persona física identificada o identificable", la obligación contenida en la norma reclamada no está en ninguno de los supuestos prohibidos tanto por la Ley Federal de Protección de Datos Personales en Posesión de Particulares, como en la propia legislación local, e incluso de la Ley Federal de Acceso a la Información (vigente al momento).

En este contexto, y bajo el entendido que el dato de carácter personal es aquella información numérica, alfabética, gráfica, fotográfica acústica o de cualquier otro tipo concerniente a personas físicas, el supuesto concreto aborda un tema de información alfabética, pero el supuesto normativo prohibitivo nos e actualiza, toda vez que la persona identificada es aquella cuya identidad está determinada, mientras que la persona identificable es aquella cuya identidad pueda determinarse, ya sea directa o indirectamente mediante cualquier información referida a 
su identidad física, fisiológica, psíquica, económica, cultural o social.

Dicho lo anterior, la exhibición del tipo de "seguridad social" que se ofrece a la plantilla de la Notaria no recae en ninguna prohibición sobre datos personales, toda vez que no se está tampoco afectando la esfera íntima del titular, toda vez que su utilización o conocimiento de parte de la sociedad no puede dar lugar en ningún momento a discriminación, o que conlleve un grave riesgo para estos. En concreto, la obligación de informar no incluye en ningún momento la revelación de aspectos tales como el origen racial o étnico, estado de salud presente y futuro, información genética, creencias religiosas, filosóficas y morales, afiliación sindical, opiniones políticas, o preferencias sexuales.

Sentado lo anterior, siguiendo el test de proporcionalidad en su primera fase, la publicación de la plantilla del personal y el tipo de seguridad social que se les ofrece, encuentra asidero constitucional en el ejercicio de la institución de la función notarial, así como la existencia de un marco constitucional que protege y equilibra también el ejercicio legítimo de los datos personales, ya que no resulta vulnerado tanto en su contenido esencial como en su periferia los mismos.

Evaluación de la necesidad y proporcionalidad de la medida sobre el instrumento para difundir la información

El carácter adicional de la información pública de oficio es la proactividad, es decir, que el sujeto obligado ponga a disposición de cualquier interesado en su página de internet la información. En tal sentido, para analizar si el medio que exige la ley para cumplir con el derecho a la información es válido o constituye una carga desproporcional e irrazonable para los notarios en su carácter de sujetos obligados indirectos, es necesario evaluar si existen medios alternativos para cumplir con la ratio del cuerpo normativo aprobado por el legislador Coahuilense (subprincipio de necesidad).

En la doctrina esta fase hermenéutica (Dworkin, 2009) del principio de proporcionalidad (necesidad), implica que las medidas es decir, las medidas deben ser necesarias para asegurar la obtención de los fines que fundamentan la restricción constitucional, es decir, no basta que la restricción sea en términos amplios útil para la obtención de esos objetivos (efecto útil de las acciones afirmativas), sino que debe ser la necesaria para su realización, lo que significa que el fin buscado por el legislador no se pueda alcanzar razonablemente por otros medios menos restrictivos.

Dicho esto la cuestión a dilucidar es: ¿representa una carga desproporcional o irrazonable para el notario la difusión en su página de internet de la obligación señalada en la norma? En la exposición de motivos de la Ley de Acceso a la Información la ratio que inspira dicho ordena- 
miento jurídico es una ida vanguardista, que toma en serio el derecho a la información y la protección de datos personales, pero sobre todo se encuentra basada en los principios de Gobierno Abierto, bajo el entendido que las personas gozan como derecho fundamental solicitar, difundir, investigar y recabar información pública.

\section{3.- METODOLOGÍA}

Como se mencionó en la introducción, esta investigación es de carácter cualitativa y el enfoque es documental. De acuerdo con Pino Milona (1992), este enfoque resulta útil para concluir, basado en evidencias documentadas, una interpretación y síntesis del texto a analizar. En este caso, se analiza la sentencia y los fundamentos legales que son objeto de análisis.

\section{4.-CONCLUSIONES}

La Ley de Acceso a la Información en el Estado es la primera en el país que aborda desde esta visión moderna este eje rector de gobierno abierto. Sentada esta premisa es importante manifestar que la cantidad de información que se pudiera generar con motivo de las obligaciones prescritas por el legislador local hacia el notario como sujeto obligado indirecto encuentran perfecta armonía con la disponibilidad de recursos tecnológicos con los que pueda contar el mismo, pero sobre todo, la proporcionalidad de la medida se basa en que la información que se difunda sólo será en proporcional a su carga de trabajo.

Afirmar que la información que se exige obligatoriamente por ley desbordaría su capacidad es infundado, toda vez que solamente se exige proporcionalmente lo que hace, no más, es decir, mientras más carga de trabajo exista, mayor cantidad de información pública generará, pero esto encuentra justificación en razón del interés que guarda la propia información pública, ya que es importante para la sociedad el conocerla, tal y como ha quedado apuntado previamente.

Por tanto, el mejor medio para dar difusión en la actualidad a la información pública es, sin duda alguna internet, ya que a través de esta plataforma digital se pueden dar a conocer de manera inmediata la información pública, lo cual resulta razonablemente comprensible toda vez que las distancias en el Estado de Coahuila entre cada municipio son considerablemente amplias, por lo que el tiempo que le llevaría a un particular por ejemplo, revisar si existen actos jurídicos que derivan de protocolizaciones de todos los notarios públicos en la Entidad sería demasiado tardado y ello sí representaría una carga injustificada y desproporcional hacia el titular del derecho a la información que es la sociedad en su conjunto. Con el propósito de armonizar ambos principios es plausible exigir esa medida al notario de poner a disposición del público la información pública en su página de internet, toda vez que es la mejor garantía hoy en día para garantizar el acceso efectivo a la información pública.

Revista Política, Globalidad y Ciudadanía, Vol. 6. No. 12, Julio - Diciembre 2020, Universidad Autónoma de Nuevo León, Monterrey, México, ISSN 2395-8448. 207-224. http://revpoliticas.uanl.mx/index.php/RPGyC/article/view/155 
Pareciera que el tema del derecho a la información es un tópico agotado en cuanto a sus planteamientos epistémicos y axiomáticos, no obstante, los elementos de ponderación y el análisis semiótico de la justicia constitucional constituyen nuevas voces científicas a las cuales los investigadores deberán de dar causes procedimentales, a efectos de establecer mecanismos idóneos al nuevo constitucionalismo crítico contemporáneo que garantice el actuar de las instituciones del Estado en aras - como lo sostuviera Zagrebelsky- de reconceptualizar de forma dúctil el cánon constitucional.

\section{REFERENCIAS}

Alexy, R. (1991). Teoría de la argumentación jurídica. España: Centro de Estudios Constitucionales.

Asensi Sabater, J. (1998). La época constitucional. España: Tirant lo Blanch.

Atienza Rodríguez, M. (2013). Curso de argumentación jurídica. España: Editorial Trotta.

Carbonell, M. (2008). El régimen constitucional de la transparencia, México: IIJ-UNAM

Contradicción de Tesis 24/2003-SS, (julio de 2003). Entre las sustentadas por el Primer y

Segundo Tribunales Colegiados, Ambos en materia administrativa del Tercer Circuito, Tomo XVIII, Amparo directo 193/98. Localización: [J]; 9 á Época; Segunda Sala, S.J.F.

Del Pilar Hernández, M. y Morales Sánchez, J. M. (2015). Comentario al artículo 121 constitucional, en Derechos del pueblo mexicano. México a través de sus constituciones, Senado de la República, México: Miguel Ángel Porrúa.

Dworkin, R. (2009). Los derechos en serio. España: Editorial Ariel.

Sala de la Suprema Corte de Justicia de la Nación (2012). DERECHOS FUNDAMENTALES. SU VIGENCIA EN LAS RELACIONES ENTRE PARTICULARES. Localización: [J]; 10a. Época; 1a. Sala; S.J.F. y su Gaceta; Libro XIII, Octubre de 2012; Tomo 2; 1a./J. 15/2012 (9a.).

Gaceta (20 de diciembre de 2012). Dictamen de las Comisiones Unidas de Puntos Constitucionales; de Estudios Legislativos Primera; de Gobernación y de Anticorrupción y Participación Ciudadana en materia de transparencia. No. 77. Cámara de Senadores, México, D.F. 
Islas L., J. (2007). La información pública y la jurisprudencia internacional, en Carbonell, M. y Bustillos Roñequi, J. (Coords.). Hacia una democracia de contenidos: la reforma constitucional en materia de transparencia, Instituto de Investigaciones Jurídicas (IIJ)Universidad Nacional Autónoma de México (UNAM), México,

Leal Espinoza, J. L. y López Sánchez, R. (2019) El derecho a la información y datos personales en México: una visión comaprada con el sistema interamericano y europeo de derechos humanos. España: Dykinson.

López Sánchez, R. (2016). Interpretación constitucional de los Derechos Fundamentales (Nuevos modelos hermenéuticos y argumentativos, México: Porrúa

NOTARIOS. ACTAS FUERA DE PROTOCOLO. VALOR PROBATORIO (LEGISLACIÓN

DEL ESTADO DE COAHUILA). Localización: [J]; 9a. Época; T.C.C.; S.J.F. y su Gaceta; Tomo V, Mayo de 1997. VIII.2o. J/13.

Pino Molina, M. (1992). El resumen documental: principios y métodos. Madrid: Pirámide Pleno de la Suprema Corte de Justicia de la Nación (octubre de 2013). Auditorías Ambientales Voluntarias. La información y documentación generadas por los particulares o sus auditores y entregadas a la Procuraduría Federal De Protección al Ambiente durante su tramitación, si bien son de carácter público, no podrán divulgarse si se actualizan los supuestos para su reserva temporal o se trata de datos confidenciales. Localización: [J]; 10a. Época; Pleno; S.J.F. y su Gaceta; Libro XXV; Tomo 1. P./J. 26/2013 (10a.);

Real Academia Española (2016). Voz: "Fe Pública”, Diccionario del Español Jurídico, Santiago Muñoz Machado (Director), Real Academia Española, Madrid: Consejo General del Poder Judicial de España.

Real Academia Española (2016). Voz: "Fe Pública Notarial”, Diccionario del Español Jurídico. Santiago Muñoz Machado (Director), Real Academia Española. Madrid:Consejo General del Poder Judicial de España,

Sartori, G. (2016). Ingeniería constitucional comparada. México: Fondo de Cultra Económica. Zagrebelsky, G. (2003). El derecho dúctil. Ley, derechos y justicia. España: Trotta. 